\title{
Ecología de la polinización de tres especies de Oenocarpus (Arecaceae) simpátricas en la Amazonia Colombiana
}

\author{
Luis Alberto Núñez A. ${ }^{1,2}$, Carolina Isaza ${ }^{2} \&$ Gloria Galeano ${ }^{2}$ \\ 1. Programa de Biología Universidad de La Salle, Colombia; lanunez@unisalle.edu.co \\ 2. Grupo de Investigación en Palmas Silvestres Neotropicales, Instituto de Ciencias Naturales, Universidad Nacional de \\ Colombia; caisazaar@unal.edu.co, gagaleanog@unal.edu.co
}

\author{
Recibido 20-I-2014. Corregido 10-X-2014. Aceptado 06-XI-2014.
}

\begin{abstract}
Pollination ecology of three sympatric species of Oenocarpus (Arecaceae) in the Colombian Amazon. The understanding of pollination mechanisms is vital for developing management and conservation actions of economically important species. In order to understand the pollination mechanisms of the promising palms in the genus Oenocarpus (Arecaceae), we studied floral morphology and biology, of three sympatric species in the Colombian Amazon: O. bataua, O. balickii and O. minor. During the period 2010-2012 we made direct and continuous observations of inflorescences (visitors, pollinators, and reproductive success) of the three species in every development phase. We determined the association of the palms with their floral visitors through a complex or interaction network, whereas specificity or preference of the insects for each individual palm was assessed through paired similarity analysis, similarity analysis (ANOSIM), and ordering analysis based on nonmetric multidimensional scaling (NMSD). The three species flowered throughout the year; their inflorescences have long rachillae that hang close to each other from a short rachis, and they bear flowers in dyads or triads. Inflorescences are protandrous, thermogenic; anthesis takes place during daytime but pollination is nocturnal. We recorded 79 species of insects, mainly beetles, 33 of which visited $O$. balickii, 63 visited $O$. bataua, and 33 visited $O$. minor. Although they shared some visitors, their abundance during the pistillate phase, as well as their pollen loads showed that only a few species of Curculionidae and Nitidulidae are the principal pollinators of the three studied species. Differences in network structure between staminate and pistillate phases, as well as difference in abundance found with the ANOSIM and NMSD similarity tests, suggest a high specificity of pollinators, leading to reproductive isolation among the three species. Because all pollinating beetles were found to develop their life cycles within the inflorescences, we hypothesize the occurrence of a specialized system of mutual dependence between each of this three Oenocarpus species and their pollinators. This specialization assures a year-round availability of the pollinators. Rev. Biol. Trop. 63 (1): 35-55. Epub 2015 March 01.
\end{abstract}

Key words: Coleoptera, fruitset, mutualism, tropical palms, reproductive biology.

El género Oenocarpus está conformado por nueve especies, distribuidas desde el sur de Centro América hasta el norte de Sur América, con límite en su distribución al sur en los bosques montanos de Bolivia (Henderson, Galeano, \& Bernal, 1995). El centro de origen del género es el bosque amazónico y la mayor concentración de especies se encuentra en la Amazonia Colombiana, donde se presentan siete de las nueve especies del género (Galeano, \& Bernal, 2010).
Todas las especies de Oenocarpus son palmas de gran importancia ecológica y económica (Balick, 1992; Bernal, Galeano, \& Henderson, 1991; Henderson, 2002). En los ecosistemas donde se encuentran, los frutos son la base de la dieta de gran cantidad de especies de vertebrados (Zona, \& Henderson, 1989). Los frutos son ampliamente utilizados por varias etnias y grupos humanos para la elaboración de bebidas con alto contenido proteico a partir de la pulpa (Macía et al., 2011), de la 
cual también se extrae aceite de alta calidad (Balick, 1982). Aunque predomina el consumo doméstico, en varias poblaciones de la cuenca amazónica, los frutos de especies de Oenocarpus principalmente de $O$. bataua, $O$. minor y $O$. bacaba son comercializados en los mercados locales (Brokamp et al., 2011).

Los trabajos en biología reproductiva de palmas con potencial económico han aumentado en los últimos años (Barfod, Hagen, \& Borchsenius, 2011; Fava, Covre, \& Sigrist, 2011; Núñez, \& Carreño, 2013; Núñez, \& Rojas, 2008); sin embargo, en la mayoría de los casos se han concentrado en una sola especie o varias especies no emparentadas (e.g., Fava et al., 2011; Núñez, Bernal, \& Knudsen, 2005), y son más pocos los trabajos que evalúan aspectos reproductivos de especies simpátricas congenéricas. Listabarth (1993) comparó la polinización de tres especies de Geonoma en Perú; Borchsenius (1993) comparó la polinización en tres especies de Aiphanes en Ecuador; Ervik, Tollsten, \& Knudsen (1999) la polinización de tres especies de Phytelephas en Ecuador y Henderson, Pardini, Dos Santos Rebello, Vanin y Almeida (2000) la polinización de diez especies de Bactris en la Amazonia brasilera. En dichos estudios, los principales resultados indican asociación de polinizadores particulares a cada género de palmas; sin embargo no especifican si se comparten los polinizadores entre las especies congenéricas evaluadas.

Los estudios de biología reproductiva de especies congenéricas que crecen en simpátria son importantes porque permiten conocer las estrategias de reproducción y los mecanismos de aislamiento reproductivo entre especies emparentadas (Briggs, \& Walters, 1997), ayudan avalidar los mecanismos involucrados en la especiación simpátrica que lleva a la diversificación de plantas y sus polinizadores (Herrera, 2005), permiten determinar el grado de asociación con visitantes florales y con ellola dependencia, especificidad, especialización e interferencia reproductiva a través de la competencia por polinizadores (Pellmyr, 2002), conocimientos que son fundamentales para determinar el grado de vulnerabilidad y salud de las poblaciones de plantas en su hábitat natural (Vázquez, \& Aizen, 2004).

Por otro lado, el aprovechamiento de los frutos de especies de Oenocarpus se realiza, en la mayoría de los casos, de manera destructiva, derribando las palmas productivas, práctica que ha generado una disminución considerable de las poblaciones naturales. Dentro de las estrategias para evitar el deterioro de las poblaciones en su hábitat natural pero asegurando un aprovechamiento sostenible, se destaca el desarrollo de planes de manejo (Miranda, Montaño, Zenteno, Nina, \& Mercado, 2008). En la formulación de dichos planes, especialmente para aquellas especies de palmas con potencial económico por sus frutos, se debe conocer con profundidad la biología reproductiva y polinización, con el fin de asegurar una provisión del recurso y de la regeneración poblacional.

Por todo lo anterior, el objetivo de este trabajo fue comparar la ecología de la polinización de las palmas simpátricas Oenocarpus balickii F. Kahn, O. bataua Mart. y O. minor Mart., tres palmas útiles de los bosques de la Amazonia Colombiana. Se pretendió responder las siguientes preguntas: i. ¿Existen diferencias en la morfología y biología floral de estas especies de Oenocarpus?, ii. ¿Cuál es el mecanismo de polinización en cada una de las especies?, iii. ¿Cómo está organizado el ensamblaje de visitantes florales, especialmente relacionado con la especificidad, papel en la polinización y posibilidad de transferencia intraespecífica de polen?

\section{MATERIALES Y MÉTODOS}

Área de estudio: Las observaciones de campo se realizaron entre 2010-2012, durante cuatro salidas de campo, con separación de 5-6 meses entre ellas (noviembre 2010, abril 2011, octubre 2011 y abril 2012), y con una duración de 8 a 12 días cada una. El estudio se realizó en la Estación Biológica El Zafire, ubicada a $80 \mathrm{~m}$ de altitud, en el extremo sur de la Amazonia colombiana, en el municipio de Leticia, Departamento de Amazonas (Coordenadas geográficas: $\left.3^{\circ} 46^{\prime} 39^{\prime \prime} \mathrm{S}-70^{\circ} 18^{\prime} 09^{\prime \prime} \mathrm{W}\right)$. La 
precipitación promedio anual es de $3400 \mathrm{~mm}$, con un período seco de junio a septiembre y una estación lluviosa de octubre a mayo. La temperatura promedio mensual es de $26^{\circ} \mathrm{C}$ y la humedad relativa promedio de $86 \%$. La zona presenta untipo de bosque muy Húmedo Tropical y la mayor parte del área incluye bosques de tierra firme o de altura, que crecen sobre una topografía de pendientes moderadas a altas y presentan grandes árboles, lianas y palmas (Galeano, Peñuela-Mora, \& Núñez, 2013).

\section{Especies de estudio}

Oenocarpus balickii: Es una palma monocaule de 6 a14m de alto, que crece en bosques de tierra firme por debajo de $200 \mathrm{~m}$, en el Occidente de la Amazonia, desde Venezuela hasta Perú. En Colombia sólo se presenta en el suroriente amazónico, entre los municipios de la Pedrera y Leticia (Galeano, \& Bernal, 2010).

Oenocarpus bataua: Es una palma monocaule de 7 a $20 \mathrm{~m}$ de alto. Se encuentra ampliamente distribuida en todos bosques de tierras cálidas y húmedas de los países de la región tropical suramericana, desde el nivel del mar hasta poco más de $1000 \mathrm{~m}$ (Galeano, \& Bernal, 2010).

Oenocarpus minor: Es una palma cespitosa, con 3-10 tallos de hasta $17 \mathrm{~m}$ de alto, ampliamente distribuida en el norte de Sur América (Venezuela, Ecuador, Perú, Brasil y Bolivia) hasta Centro América (Panamá y Costa Rica). Crece en zonas húmedas a plufrascoes desde el nivel del mar hasta poco más de 1 000m (Galeano, \& Bernal, 2010; Henderson et al., 1995). En Colombia se encuentra en casi todos los bosques húmedos y cálidos del país.

Morfología floral: En 20 inflorescencias de $O$. bataua, 10 inflorescencias de $O$. balickii y en 15 de $O$. minorse evaluó la morfología de las inflorescencias utilizando las siguientes variables: 1) altura de la inflorescencia, 2) número de raquilas por inflorescencia, 3) número de flores masculinas y femeninas por raquila, 4) número total de flores por inflorescencia, y 5) tamaño de las flores masculinas y femeninas.

Fases florales y biología floral: En cada especiese registró el número de inflorescencias que produjo cada palma y se determinaron las fases florales que ocurren en cada inflorescencia; las variables tenidas en cuenta fueron: hora de apertura de la yema, duración total de la floración en la inflorescencia, duración, dirección y ritmo diario de antesis de los botones florales. En las flores se registró la hora de antesis, la longevidad floral, la presencia de recompensas florales, la receptividad estigmática, la hora de la presentación y viabilidad del polen y se midió la termogenésis. La receptividad estigmática se evaluó mediante observación directa de exudados, pruebas de actividad enzimática con Peróxido de Hidrógeno (Kearns, \& Inouye, 1993) y test de colorimetría con Perex-Test de Merck (Dafni, 1992). Para determinar la viabilidad del polen se realizó una prueba de colorimetría con Perex Test de Merck (Dafni, 1992). Todas las pruebas se realizaron cada 12 horas, durante ocho días en las flores masculinas y durante toda la fase femenina en cada una de las tres especies de palmas. La termogénesis fue medida en cinco inflorescencias en fase masculina y cinco en fase femenina de cada palma, con termómetros digitales Cox Technologies, Inc. Se introdujo el sensor de los termómetros y registramos la temperatura interna de la inflorescencia, la cual fue comparada con la temperatura del ambiente medida de manera simultánea con otro termómetro.

Visitantes florales: Debido a que las tres especies estudiadas presentaron protandria, se evaluó la composición, riqueza, grado de interacción de los visitantes y rol en la polinizaciónen cada fase (i.e., masculina y femenina) para cada especie. Para conocer los grupos de insectos que visitan las inflorescencias de las especies de Oenocarpus, se realizaron colectas en 11 diferentes inflorescencias de $O$. bataua ( 6 en fase masculina y 5 en fase femenina), 10 inflorescencias de $O$. balickii (5 por cada 
fase) y 11 de $O$. minor (6 en fase masculina y 5 en fase femenina). Cada una de las inflorescencias fue embolsada, y con fuertes sacudidas se logró que los insectos cayeran dentro de la bolsa. Este procedimiento se repitió de manera consecutiva durante los tres primeros días de la floración en cada inflorescencia, período donde se presentó la mayor actividad de los visitantes en cada fase. La abundancia relativa de cada insecto visitante se calculó como la suma de cada colecta durante los tres días. Cada insecto visitante fue montado, etiquetado, fotografiado y evaluada su presencia en cada palma. La identificación se realizó hasta el nivel taxonómico posible y algunos insectos fueron enviados a especialistas para su identificación o confirmación. Se generó una colección de referencia que se depositó en la colección entomológica del Instituto de Ciencias Naturales (ICN) de la Universidad Nacional de Colombia, en Bogotá.

Definición de polinizadores: El papel de cada visitante en la polinización de las tres especies de Oenocarpus, se determinó a partir de un análisis de cantidad (abundancias) de insectos que llegaron a las flores en fase femenina y cantidad de polen transportado en el cuerpo de cada insecto. Siguiendo la metodología de Núñez et al. (2005), la calidad en la polinización se evaluó a partir del conteo de cargas polínicas de una muestra de 10 individuos de cada especie de insecto que llegó durante la fase femenina. La presencia de polen heteroespecífico en el cuerpo de los insectos se comprobó comparando el tipo de polen encontrado con una muestra de polen previamente colectado en flores de cada una de las tres palmas.

Grado de asociación de visitantes florales: Para determinar la asociación interespecífica de los insectos visitantes con las tres palmas se utilizó una red compleja o red de interacción. La red permite conocer la fuerza de la asociación (número de enlaces), la estructura, dinámica y función de la comunidad de visitantes asociados con las inflorescencias (Guimarães et al., 2007). Construimos una matriz cualitativa $\left(\mathrm{P}^{*} \mathrm{~V}_{\mathrm{m}}, \mathrm{P}^{*} \mathrm{~V}_{\mathrm{f}}\right)$ entre las palmas $(\mathrm{P})$ y los insectos visitantes $(\mathrm{V})$, tanto para la fase masculina $\left(\mathrm{V}_{\mathrm{m}}\right)$ y la fase femenina $\left(\mathrm{V}_{\mathrm{f}}\right)$, como para los polinizadores $\left(\mathrm{P}^{*} \mathrm{~V}_{\mathrm{p}}\right)$. En las matrices, la interacción se representa con 1 cuando ocurre y 0 cuando es ausente. Para cada matriz se calcularon los principales parámetros cualitativos y cuantitativos de la red (estructura de la red, ensamblaje, grado de anidamiento, conectancia y grado de asociación). Los parámetros fueron calculados con las librerías Bipartite, Vegan y Network (Dormann, Frund, Blutgeb, \& Gruber, 2009) con software R (ver. 2.11.1, R Development CoreTeam 2010).

Especificidad: La especificidad o preferencia de los insectos por cada palma se evaluó con un análisis de similaridad pareada, un análisis de similitudes (ANOSIM) y un análisis de ordenamiento con la técnica de escalamiento multidimensional no métrico (NMSD) (Cox \& Cox, 2001).Cada análisis se realizó para cada fase y para los insectos definidos como polinizadores. El análisis de similaridad pareada se realizó a partir de datos de presencia-ausencia de cada visitante y como resultado se generó una matriz pareada que indica el valor del índice de similaridad entre cada par de especies. Los valores cercanos a 0 indicaron baja similaridad en composición de visitantes y los cercanos a 1 dan prueba de que las palmas comparadas comparten sus visitantes florales.

Para el análisis de similitudes (ANOSIM) y el escalamiento multidimensional no métrico (NMSD) se construyeron matrices a partir de las abundancias relativas de cada visitante. La prueba (NMSD) generó una figura en dos dimensiones que agrupa o separa las especies de acuerdo al grado de similaridad. El ANOSIM es una prueba de similitud no paramétrica de una vía que define si hay diferencias significativas de los visitantes entre las palmas, en términos de abundancia de las especies de visitantes. La prueba ANOSIM produce un estadístico $\mathrm{R}$, que es una medida absoluta de distancia o similaridad entre las palmas. Los valores cercanos a 1 indican baja similitud entre los visitantes por fase, mientras que los valores cercanos a 0 indicaron alta similitud 
entre los visitantes por fase entre las especies (Hammer, Harper, \& Ryan, 2001). Como resultado se muestra el valor de $\mathrm{R}$ para cada caso: para fase masculina $R_{m}$, para fase femenina $\mathrm{R}_{\mathrm{f}}$, y para insectos con cargas polínicas $\mathrm{R}_{\mathrm{p}}$. La similaridad pareada, el ANOSIM y el NMSD se realizaron con el programa PAST $^{\circledR}$ (Clarke, \& Gorley, 2001).

Éxito reproductivo: Comparamosel éxito reproductivode cada palma a través de la relación: total de flores producidas por inflorescencia y la cantidad de frutos que se desarrollan y maduran. Se cortaron 10 infrutescencias con frutos maduros por cada especie de palma y se contó el número total de frutos que produjo cada infrutescencia. En las mismas infrutescencias se contaron todas las cicatrices de flores y con ello se estableció el número total de flores formadas por raquila y por inflorescencia. El éxito reproductivo se calculó como el porcentaje de frutos producidos según la fórmula propuesta por Dafni (1992): total de frutos formados/total de flores producidas*100. Las flores que no se desarrollaron en frutos se denominaron abortos y la tasa de abortos en cada especie se obtuvode la diferencia entre el total de flores producidas $\mathrm{y}$ el total de frutos formados.

\section{RESULTADOS}

Morfología floral: Las palmas consideradas en este estudio son similares en la morfología de sus inflorescencias (Fig. 1A, Fig. 1B y Fig. 1C), y se ubican en el mismo estrato del bosque desde 2.7 a $15 \mathrm{~m}$. Los aspectos reproductivos diferenciables entre las tres palmas se presentan en el cuadro 1. Las inflorescencias tienen la forma de una cola de caballo, son de un color crema al inicio de la antesis; se vuelven pardas después de la apertura de la bráctea peduncular y luego de la fecundación de sus flores adquieren una coloración amarillo ocre. Aunque a nivel de la inflorescencia las tres especies tienen la misma configuración estructural, hay diferencias de tamaño, forma y color de los botones florales (Fig. 1).
En las tres especies estudiadas las inflorescencias consisten de un raquis corto y resistente, del cual penden raquilas o ramas simples insertadas lateral y adaxialmente (Fig. 1A, Fig. 1B y Fig. 1C). La distribución de flores en las raquilas se presenta en tríadas en la parte proximal, y en díadas hacia la parte apical de la raquila. Cada tríada está conformada por una flor central femenina y dos laterales masculinas, mientras las díadas están conformadas por dos flores masculinas (Fig. 1D, Fig. 1E y Fig. 1F). La cantidad de tríadas es diferente en cada palma: en $O$. bataua y $O$. minor se pueden encontrar hasta la parte media de la raquila, mientras que en $O$. balickii se pueden extender a toda la raquila; también es frecuente observar algunas raquilas con pocas tríadas y con predominio de díadas a lo largo de la raquila. Ocasionalmente, $O$. minor presentó inflorescencias con flores exclusivamente masculinas.

Fases florales y biología floral: En cada una de las visitas a las poblaciones fue posible encontrar yemas, flores y frutos en diferentes estados de desarrollo en las tres especies, lo que sugiere floración continua en las tres palmas. El número de inflorescencias que produce cada individuo varió entre especies: $O$. bataua produjo entre 1-2, $O$. balickii entre 1-3 y 1-5 en $O$. minor. El desarrollo de las inflorescencias parece ser consecutivo, debido a la presencia, en un mismo individuo, de infrutescencias en diferente grado de desarrollo de sus frutos; no obstante, en $O$. minor se puede presentar desarrollo simultáneo de inflorescencias debido a su hábito cespitoso.

Una vez se ha completado el desarrollo de cada inflorescencia, la bráctea peduncular que la envuelve abre, cae, deja expuestas las raquilas, e inicia el ciclo reproductivo en el que se presentan cinco etapas, morfológica y funcionalmente diferenciables, pero son similares en las tres especies de Oenocarpus estudiadas: 1. Apertura y liberación de la bráctea que recubre la inflorescencia; se exponen los botones de las díadas y tríadas e inmediatamente algunas flores masculinas inician la antesis. 2. Antesis de las flores masculinas; hay 

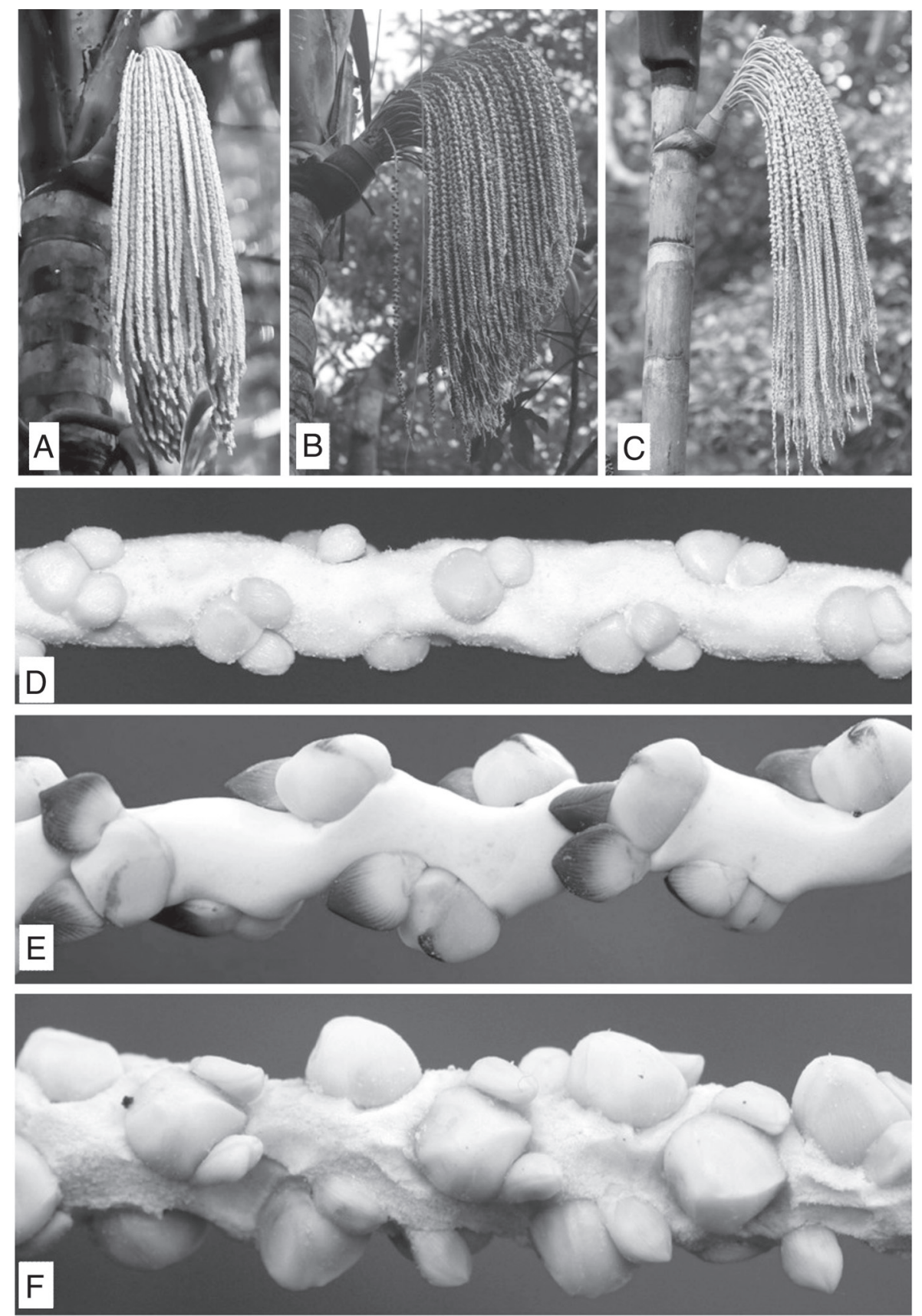

Fig. 1. Aspectos de las inflorescencias y raquilas de tres especies de Oenocarpus. A y D. O. balickii, B y E. O. bataua, y C y F. O. minor.

Fig. 1. Aspects of the inflorescences and rachillae of three Oenocarpus species. A, D. O. balickii, B, E. O. bataua and C, F. O. minor. 


\section{CUADRO 1}

Caracteres reproductivos de las especies de Oenocarpus en la Amazonia colombiana. Se presenta el promedio \pm desviación estandar yel tamaño de la muestra en paréntesis

TABLE 1

Reproductive characteres of the Oenocarpus species in the Colombian Amazon. The mean, standard deviation and sample size in parentheses

\begin{tabular}{lccc}
\multicolumn{1}{c}{ Carácter } & O. balickii & O. bataua & O. minor \\
Altura de inflorescencia (m) & $11.4 \pm 3.2(10)$ & $13.6 \pm 2.2(20)$ & $9.8 \pm 2.2(15)$ \\
Inflorescencias/individuo & $2 \pm 1(17)$ & $1 \pm 1(25)$ & $4 \pm 1(15)$ \\
Longitud raquilas (cm) & $54.4 \pm 5.2(100)$ & $95 \pm 8.2(100)$ & $56.2 \pm 7.2(100)$ \\
Raquilas/inflorescencia & $91 \pm 15(100)$ & $195 \pm 37(100)$ & $93 \pm 27(100)$ \\
Flores masculinas/raquila & $299 \pm 61(100)$ & $379 \pm 54(100)$ & $343 \pm 68(100)$ \\
Flores femeninas/raquila & $44 \pm 37(100)$ & $48 \pm 25(100)$ & $44 \pm 17(100)$ \\
Flores masculina/inflorescencia & $27209 \pm 9609(10)$ & $73905 \pm 609(10)$ & $31899 \pm 2609(10)$ \\
$\begin{array}{l}\text { Flores femenina/inflorescencia } \\
\text { Éxito reproductivo: Total frutos/ } \\
\text { total flores formadas (\%) }\end{array}$ & $4004 \pm 501(10)$ & $9360 \pm 1501(10)$ & $4092 \pm 1501(10)$ \\
$\begin{array}{l}\text { Aborto: Total frutos/total flores } \\
\text { formadas (\%) }\end{array}$ & 45 & 21 & 21 \\
\hline
\end{tabular}

exposición de anteras y liberación de polen y a las pocas horas caen las flores masculinas en antesis. 3. Fase inactiva, que ocurre cuando ya han caído todas las flores masculinas, y en cada raquila tan sólo quedan los botones de las flores femeninas. 4. Desarrollo y antesis de las flores femeninas, donde los estigmas emergen completamente; el color blanco y la presencia de una sustancia hialina sobre la superficie de los estigmas son prueba de su viabilidad; es en esta fase cuando ocurre la polinización, y 5. Desarrollo de frutos, que se produce con el crecimiento del ovario, luego de la fertilización; con el paso de los días toma la forma característica del fruto de cada especie, y luego de 8 a 12 meses los frutos maduran y se inicia su caída de la infrutescencia o la remoción por parte de animales.

Aunque las inflorescencias de las tres especies presentan modelo similar de floración, con una fase inicial masculina, una fase intermedia inactiva y la fase final femenina, en cada especie varió el tiempo en el que permanecieron funcionales. Las inflorescencias de $O$. bataua fueron funcionales durante 30-32 días, las de $O$. balickii entre 23-25 días y las de $O$. minor entre 25-27 días. En todos los casos, la fase masculina fue la más larga y la que presentó mayor variación entre especies. La fase masculina se extendió hasta por 15 días en $O$. bataua, 14 días en $O$. balickii y 9-10 en $O$. minor. Luego de la fase masculina, las inflorescencias pasaron por una fase inactiva que tardó entre 5 a 8 días en todas las especies, en donde los botones de las flores femeninas completaron su desarrollo. La fase femenina fue la etapa final del ciclo reproductivo en cada inflorescencia; tardó entre 1 a 4 días en las tres especies, pero fue durante el primero y segundo día cuando se presentó el mayor número de flores con los estigmas receptivos.

La biología floral de las tres especies siguió un mismo patrón: fueron protandras, termogénicas, y liberaron aroma perceptible y diferenciable entre especies. Las inflorescencias en fase masculina proporcionaron gran cantidad de polen como recompensa, mientras que las femeninas no produjeron ningún tipo de recompensa. Los eventos que ocurrieron durante el ciclo floral y que fueron comunes en las tres especies son: la bráctea peduncular abre a lo largo del día y deja expuestos los botones florales. Un primer grupo de flores masculinas inician antesis al final de la tarde del primer 
día y entre 8-12 horas después dejan de ser funcionales y caen. En la tarde del siguiente día un nuevo grupo de flores masculinas entra en antesis; y así cada día hasta terminar la fase, nuevas flores abren y caen de la inflorescencia. La presencia de fuerte aroma siempre fue notoria cuando las flores estuvieron en antesis y coincidió con el aumento de la temperatura interna de las inflorescencias que alcanzó en promedio $4^{\circ} \mathrm{C} \pm 2(\mathrm{DE}, \mathrm{n}=5)$ por encima de la temperatura ambiente en fase femenina $y$ $5.2^{\circ} \mathrm{C} \pm 1.3(\mathrm{DE}, \mathrm{n}=5)$ en fase masculina en O. bataua; $7^{\circ} \mathrm{C} \pm 1(\mathrm{DE}, \mathrm{n}=5)$ por encima de la temperatura ambiente en fase femenina y $6.2^{\circ} \mathrm{C} \pm 1.3$ (DE, $\mathrm{n}=5$ ) en fase masculina en $O$. minor; y $5.5^{\circ} \mathrm{C} \pm 2.3$ (DE, $\mathrm{n}=5$ ) por encima de la temperatura ambiente en fase femenina y $4.8^{\circ} \mathrm{C} \pm 1.7(\mathrm{DE}, \mathrm{n}=5)$ en fase masculina en $O$. balickii. La viabilidad polínica se mantuvo constante a lo largo de la fase masculina: se presentaron porcentajes de viabilidad entre 65 y $80 \%$ en $O$. bataua, $48-78 \%$ en $O$. balickii, y $68-80 \%$ en $O$. minor. Por su parte, la exposición de los estigmas ocurrió a lo largo del día y según las pruebas con peróxido de hidrógeno y test de colorimetría, pueden durar receptivos hasta por dos días. En todas las especies, las flores femeninas presentaron una etapa de preantesis en la cual los estigmas emergieron completamente justo antes de entrar en antesis (Fig. 2C). Sin embargo, en algunas flores dentro de cada inflorescencia los estigmas no emergieron, no completaron su desarrollo o entraron en antesis cuando ya había pasado la polinización de la mayoría de las flores de la inflorescencia. Estas flores asincrónicas abortaron en los días siguientes.

Visitantes: Se registraron 79 especies de insectos, de las cuales 33 especies visitaron $O$. balickii, 63 visitaron a $O$. bataua y 33 especies visitaron a $O$. minor. El orden Coleoptera fue el más diverso con 45 especies, seguido de Himenoptera con 22 especies, Diptera con seis especies, Heteroptera con cinco especies y una especie de Dermaptera. Las familias con el mayor número de especies fueron: Curculionidae (25 spp.), Chrysomelidae (7 spp.), Staphylinidae (5 spp.), Scarabaeideae (4 spp.) y Nitidulidae (3 spp.) (Cuadro 2). Las abejas sociales (Hymenoptera: Meliponinae), presentaron 10 especies.

CUADRO 2

Insectos visitantes de las inflorescencias de Oenocarpus balickii, O. bataua y $O$. minor en la Amazonia colombiana. Abundancia promedio y carga de polen promedio de individuos

TABLE 2

List of inflorescence visitors of Oenocarpus balickii, O. bataua and O. minor in the Colombian Amazon. Average abundance/pollen load

\begin{tabular}{|c|c|c|c|c|}
\hline $\mathrm{N}^{\mathrm{o}}$ & ORDEN/FAMILIA/Géneros/especie & O. balickii & O. bataua & O. minor \\
\hline & COLEOPTERA & & & \\
\hline & CURCULIONIDAE & & & \\
\hline & Derelomini & & & \\
\hline 1 & Anchylorhynchus tricarinatus & 0 & $356 / 125$ & 0 \\
\hline 2 & Anchylorhynchus bicarinatus & 0 & 0 & $245 / 145$ \\
\hline 3 & Anchylorhynchus sp. 1 & $423 / 246$ & 0 & 0 \\
\hline 4 & Phyllotrox sp. 35 & $152 / 87$ & 0 & 0 \\
\hline 5 & Phyllotrox sp. 36 & 0 & $125 / 83$ & 0 \\
\hline 6 & Phyllotrox sp. 37 & $15 / 25$ & $32 / 45$ & $142 / 44$ \\
\hline 7 & Phyllotrox sp. 38 & 0 & $21 / 125$ & 0 \\
\hline 8 & Phytotribus sp. 1 & $19 / 56$ & 0 & 0 \\
\hline 9 & Phytotribus sp. 2 & 0 & $4 / 21$ & 0 \\
\hline 10 & Terires sp. 1 & 0 & $32 / \mathrm{NP}$ & 23/NP \\
\hline 11 & Terires sp. 3 & 6/NP & $15 / 234$ & 9 \\
\hline
\end{tabular}


CUADRO 2 (Continuación) / TABLE 2 (Continued)

\begin{tabular}{|c|c|c|c|c|}
\hline $\mathrm{N}^{\mathrm{o}}$ & ORDEN/FAMILIA/Géneros/especie & O. balickii & O. bataua & O. minor \\
\hline 12 & Derelomus sp. 1 & 0 & 1/NP & 0 \\
\hline 13 & Andranthobius sp. 2 & 0 & 0 & $54 / \mathrm{NP}$ \\
\hline 14 & Acalyptinae Gen. 2 sp. 1 & $12 / \mathrm{NP}$ & 193/NP & $24 / 23$ \\
\hline 15 & Perelleschus sp. 1 & 0 & $37 / 87$ & 0 \\
\hline 16 & $\begin{array}{l}\text { Perelleschus sp. } 2 \\
\text { Baridinae }\end{array}$ & 0 & $54 / 43$ & $5 / \mathrm{NP}$ \\
\hline 17 & Baridinae Gen. 1, sp. 1 & 0 & $21 / 21$ & $24 / 109$ \\
\hline 18 & Baridinae Gen. 4, sp. 1 & 0 & $543 / 234$ & 0 \\
\hline 19 & Baridinae Gen. 4, sp. 2 & $124 / 543$ & $79 / 12$ & $128 / 112$ \\
\hline 20 & Hustachea sp. 1 & 0 & $54 / \mathrm{NP}$ & 0 \\
\hline 21 & Bondariella sp. 1 & 0 & 4/NP & 0 \\
\hline 22 & $\begin{array}{l}\text { Limnonotus sp. } 1 \\
\text { Molytinae }\end{array}$ & $264 / 543$ & 6/NP & 0 \\
\hline 23 & Homalinotus histrix & 0 & $4 / \mathrm{NP}$ & 2/NP \\
\hline 24 & $\begin{array}{l}\text { Odontoderes sp. } 1 \\
\text { Rhynchophorinae }\end{array}$ & 0 & $4 / \mathrm{NP}$ & $2 / \mathrm{NP}$ \\
\hline 25 & $\begin{array}{l}\text { Metamasius hemipterus sericeus } \\
\text { NITIDULIDAE }\end{array}$ & $3 / \mathrm{NP}$ & $5 / \mathrm{NP}$ & 0 \\
\hline 26 & Mystrops salazari & $123 / 234$ & $359 / 145$ & $146 / 124$ \\
\hline 27 & Mystrops sp. 2 & 0 & $54 / 54$ & 0 \\
\hline 28 & $\begin{array}{l}\text { Gen. } 2 \text { sp. } 1 \\
\text { STAPHYLINIDAE } \\
\text { Aleocharinae }\end{array}$ & $12 / \mathrm{NP}$ & $23 / \mathrm{NP}$ & 9/NP \\
\hline 29 & Amazoncharis sp. 1 & 0 & $23 / \mathrm{NP}$ & 0 \\
\hline 30 & Aleochara sp. 1 & $23 / \mathrm{NP}$ & $154 / \mathrm{NP}$ & $25 / \mathrm{NP}$ \\
\hline 31 & Staphylininae & & & \\
\hline 32 & Xanthopygus sp. 1 & $32 / \mathrm{NP}$ & $46 / \mathrm{NP}$ & $12 / \mathrm{NP}$ \\
\hline 33 & Aleochara sp. 1 & $4 / \mathrm{NP}$ & 0 & 0 \\
\hline 34 & $\begin{array}{l}\text { Philonthus sp. } 2 \\
\text { CHRYSOMELIDAE } \\
\text { Alticinae }\end{array}$ & $12 / \mathrm{NP}$ & $26 / \mathrm{NP}$ & $13 \mathrm{NP}$ \\
\hline 35 & Longitarsus sp. 1 & 0 & $12 \mathrm{NP}$ & 6/NP \\
\hline 36 & Longitarsus sp. 2 & $5 / \mathrm{NP}$ & $7 / \mathrm{NP}$ & 4/NP \\
\hline 37 & $\begin{array}{l}\text { Gen. } 2 \text {, sp. } 1 \\
\text { Galerucinae }\end{array}$ & 0 & $4 / \mathrm{NP}$ & $2 / \mathrm{NP}$ \\
\hline 38 & Monolepta sp. 1 & $5 / \mathrm{NP}$ & $12 / \mathrm{NP}$ & $5 / \mathrm{NP}$ \\
\hline 39 & Monolepta sp. 2 & 0 & 8/NP & 0 \\
\hline 40 & Gen. 2, sp. 1 & 0 & $4 / \mathrm{NP}$ & 0 \\
\hline 41 & $\begin{array}{l}\text { Gen. } 2 \text {, sp. } 2 \\
\text { SCARABAEIDAE } \\
\text { Dynastinae }\end{array}$ & 0 & $7 / \mathrm{NP}$ & 0 \\
\hline 42 & Cyclocephala discolor & 0 & $12 / \mathrm{NP}$ & 0 \\
\hline 43 & Cyclocephala amazona & 9/NP & 0 & 0 \\
\hline 44 & Cyclocephala stictica & 0 & $14 / \mathrm{NP}$ & 0 \\
\hline 45 & $\begin{array}{l}\text { Cyclocephala guianae-endrodi } \\
\text { DERMAPTERA }\end{array}$ & 0 & $10 / \mathrm{NP}$ & 0 \\
\hline 46 & $\begin{array}{l}\text { sp. } 1 \\
\text { HYMENOPTERA }\end{array}$ & 0 & $18 / \mathrm{NP}$ & 0 \\
\hline
\end{tabular}


CUADRO 2 (Continuación) / TABLE 2 (Continued)

\begin{tabular}{|c|c|c|c|c|}
\hline $\mathrm{N}^{\mathrm{o}}$ & ORDEN/FAMILIA/Géneros/especie & O. balickii & O. bataua & O. minor \\
\hline & Meliponinae & & & \\
\hline 47 & Oxytrigona sp. 1 & $12 / 456$ & $23 / 348$ & 0 \\
\hline 48 & Partamona sp. 1 & 0 & 0 & 23/NP \\
\hline 49 & Partamona sp. 2 & 0 & 0 & $15 / \mathrm{NP}$ \\
\hline 50 & Plebeia aff. timida & 0 & $18 / \mathrm{NP}$ & 0 \\
\hline 51 & Plebeia $\mathrm{sp} .1$ & $5 / \mathrm{NP}$ & $12 / \mathrm{NP}$ & $15 / \mathrm{NP}$ \\
\hline 52 & Plebeia sp. 2 & 0 & $5 / \mathrm{NP}$ & $12 / \mathrm{NP}$ \\
\hline 53 & Plebeia sp. 3 & $8 / \mathrm{NP}$ & 0 & $10 / \mathrm{NP}$ \\
\hline 54 & Trigonisca sp. 1 & $3 / \mathrm{NP}$ & 0 & $3 / \mathrm{NP}$ \\
\hline \multirow[t]{2}{*}{55} & Trigonisca sp. 2 & 0 & 9/NP & 0 \\
\hline & HALICTIDAE & & & \\
\hline 56 & Habralictus sp. 1 & 0 & 9/NP & 0 \\
\hline \multirow[t]{2}{*}{57} & Lasioglossum sp. 1 & $3 / \mathrm{NP}$ & 0 & 0 \\
\hline & FORMICIDAE & & & \\
\hline 58 & Ectatamma tuberculatum & 0 & $3 / \mathrm{NP}$ & 0 \\
\hline 59 & Camponotus sp. 1 & 0 & $3 / \mathrm{NP}$ & 0 \\
\hline 60 & Pseudomyrmex sp. 1 & 2 & $3 / \mathrm{NP}$ & 0 \\
\hline 61 & Pseudomyrmex sp. 2 & 0 & $3 / \mathrm{NP}$ & 0 \\
\hline \multirow[t]{2}{*}{62} & Indeterminada $\mathrm{sp.} 1$ & $23 / \mathrm{NP}$ & 0 & 0 \\
\hline & BRACONIDAE & & & \\
\hline 63 & Bracon sp. 1 & 0 & $3 / \mathrm{NP}$ & 0 \\
\hline \multirow[t]{2}{*}{64} & Triaspis sp. 1 & 0 & $13 / \mathrm{NP}$ & $5 / \mathrm{NP}$ \\
\hline & ICHNEUMONOIDAE & & & \\
\hline \multirow[t]{2}{*}{65} & sp. 1 & 0 & $8 / \mathrm{NP}$ & 0 \\
\hline & FIGITIDAE & 0 & $3 / \mathrm{NP}$ & 0 \\
\hline \multirow[t]{2}{*}{66} & sp. 1 & 0 & $8 / \mathrm{NP}$ & 0 \\
\hline & VESPIDAE & & & \\
\hline 67 & Protopolybia sp. 1 & 0 & $3 / \mathrm{NP}$ & 0 \\
\hline \multirow[t]{3}{*}{68} & Polybia sp. 1 & $3 / \mathrm{NP}$ & 0 & 0 \\
\hline & DIPTERA & & & \\
\hline & DROSOPHILIDAE & $26 / \mathrm{NP}$ & $54 / \mathrm{NP}$ & $12 / \mathrm{NP}$ \\
\hline 69 & Drosophila sp. 1 & $26 / \mathrm{NP}$ & $54 / \mathrm{NP}$ & $12 / \mathrm{NP}$ \\
\hline 70 & Drosophila sp. 2 & $1 / \mathrm{NP}$ & 0 & $8 / \mathrm{NP}$ \\
\hline 71 & Syrphidae sp. 1 & 0 & $3 / \mathrm{NP}$ & 0 \\
\hline 72 & Syrphidae sp. 2 & 0 & $2 / \mathrm{NP}$ & 0 \\
\hline 73 & Indeterminada sp. 1 & $2 / \mathrm{NP}$ & 0 & 0 \\
\hline \multirow[t]{3}{*}{74} & Indeterminada sp. 2 & 0 & $5 / \mathrm{NP}$ & 0 \\
\hline & HETEROPTERA & & & \\
\hline & ANTHOCORIDAE & & & \\
\hline 75 & Xylocaris sp. 1 & $3 / \mathrm{NP}$ & $4 / \mathrm{NP}$ & 6/NP \\
\hline \multirow[t]{2}{*}{76} & Gen. 2 sp. 1 & 0 & 6/NP & 0 \\
\hline & MIRIDAE & & & \\
\hline 77 & sp. 1 & $5 / \mathrm{NP}$ & $10 / \mathrm{NP}$ & $7 / \mathrm{NP}$ \\
\hline \multirow[t]{2}{*}{78} & sp. 2 & $2 / \mathrm{NP}$ & 0 & $5 / \mathrm{NP}$ \\
\hline & THAUMASTOCORIDAE & & & \\
\hline 79 & Discocoris drakei & $56 / / \mathrm{NP}$ & 79/NP & $125 / \mathrm{NP}$ \\
\hline
\end{tabular}

$\mathrm{NP}=$ Insectos sin polen; $0=$ ausencia de visita del insecto.

$\mathrm{NP}=$ No pollen presence on insect body; $0=$ Not insects visitation. 
El patrón de abundancia de los visitantes florales en fase masculina y femenina en cada palma se caracterizó por tener pocas especies abundantes, mientras la gran mayoría de las especies tuvieron pocos individuos (Cuadro 2). Una inflorescencia de $O$. balickii a lo largo de su fase masculina fue visitada en promedio por $3390( \pm 1237 \mathrm{DE}, \mathrm{n}=5)$ insectos; una de $O$. bataua fue visitada en promedio por 7644 ( $\pm 2356 \mathrm{DE}, \mathrm{n}=6)$ insectos, y una de $O$. minor por $3327( \pm 563 \mathrm{DE}, \mathrm{n}=6)$ insectos. El número de insectos que visitaron las inflorescencias en fase femenina fue menor en todas las especies. Generalmente, fueron los mismos visitantes abundantes de la fase masculina, y no se encontró un visitante exclusivo de la fase femenina. Una inflorescencia a lo largo de la fase femenina de $O$. balickii fue visitada en promedio por 1 $130( \pm 437 \mathrm{DE}, \mathrm{n}=5)$ insectos; una de $O$. bataua en promedio por $2548( \pm 1436 \mathrm{DE}, \mathrm{n}=5)$ insectos, y una de O. minor por $1109( \pm 425 \mathrm{DE}$, $\mathrm{n}=5$ ) insectos.

La hora de llegada de la mayoría de visitantes florales en fase masculina y femenina fue crepuscular a nocturna (18:30 a 20:00 horas) y coincidió con el ritmo de apertura de los botones florales, la termogénesis y la liberación del aroma floral. A medida que las flores entraban en antesis, una nube de visitantes accedía a ellas. Esto se repetía día tras día en la medida que abrieran nuevas flores y a lo largo de todos los días que durara la fase masculina. En fase femenina los insectos llegaban solamente el primero y segundo día; igualmente llegaban a la flor en una nube pero rápidamente la abandonaban, por lo que frecuentemente la inflorescencia en fase femenina no presentaba actividad significativa de visitantes. Algunos visitantes, principalmente abejas, llegaban a las inflorescencias en fase masculina durante el día.

El comportamiento de los visitantes y polinizadores en las inflorescencias inició con la llegada de los insectos a las flores en fase masculina en busca de polen, de pareja y de lugares para ovipositar. Durante los días que duró la fase masculina y de manera constante, las inflorescencias les proporcionaron grandes cantidades de polen; luego de consumirlo buscaron pareja y copularon, para posteriormente dirigirse a un sitio ideal para ovipositar como puede ser el pedúnculo de la inflorescencia, la bráctea peduncular, las raquilas, las flores masculinas y femeninas, y los frutos que inician su desarrollo. Las larvas que se desarrollaron después de la oviposición continuaron ocultas, y completaron su ciclo de vida en las estructuras de la palma; pocos meses después emergen los adultos, que guiados por el aroma floral buscan otra inflorescencia para iniciar el ciclo nuevamente.

Red de interacción: Los principales índices cualitativos y cuantitativos de la red de interacción $(\mathrm{PxV})$ entre palmas $(\mathrm{P})$ y visitantes florales (V) se resumen en el cuadro 3. La inflorescencia de cada palma en fase masculina fue visitada en promedio por 24 especies de insectos y en fase femenina por 8 especies, de los cuales tan sólo cuatro especies (principalmente coleópteros), transportaron polen en sus cuerpos (Fig. 2). La topología de la red es diferencial entre fases de cada palma. La diversidad, el número de enlacesy el número medio de interacciones por inflorescencia disminuyeron en la fase femenina y en los insectos cargados con polen, con respecto a la fase masculina (Fig. 2, Cuadro 3).

Pocas especies de insectos fueron compartidos por todas las palmas ya que de las 79 especies de visitantes registrados y una matriz (PxV) máxima posible de 237 enlaces, se registraron tan sólo 133 enlaces en fase masculina, 48 en fase femenina y 28 con polinizadores, lo que indica que los insectos tienen preferencias por una palma en particular y la mayoría de visitantes no acceden a las flores de las otras tres palmas congenéricas, por lo cual los enlaces posibles no se completan. La preferencia de cada insecto por una palma en particular se confirma con el bajo valor en el número medio de interacciones por polinizador, que fue de tan sólo 1.0 en fase masculina y 0.89 en fase femenina (Cuadro 3). 
CUADRO 3

Índices de la Red Bipartita

TABLE 3

Network bipartite Indexes

\begin{tabular}{|c|c|c|c|}
\hline & Masculina (M) & Femenina $(\mathrm{F})$ & Polen $(\mathrm{P})$ \\
\hline Número de especies de insectos visitantes (V) & 79 & 30 & 19 \\
\hline Número de especies de palmas $(\mathrm{P})$ & 3 & 3 & 3 \\
\hline Riqueza de especies & 82 & 33 & 22 \\
\hline Tamaño de la Matriz $\left(\mathrm{P}^{*} \mathrm{~V}\right)$ & 237 & 90 & 57 \\
\hline Número total de interacciones registradas & 133 & 48 & 28 \\
\hline \multicolumn{4}{|c|}{ ÍNDICES CUALITATIVOS (Presencia / Ausencia de interacciones) } \\
\hline Número medio de interacciones por inflorescencia & 24.6 & 8.3 & 4 \\
\hline Número medio de interacciones por polinizador & 1.0 & 0.89 & 0.742 \\
\hline Conectancia & 0.5 & 0.5 & 0.4 \\
\hline C-score Insectos & 0.42 & 0.674 & 0.538 \\
\hline C-score Palmas & 0.4 & 0.3 & 0.3 \\
\hline Grado de anidamiento & 25.0 & 27.7 & 32.1 \\
\hline Peso de Anidamiento & 0.4 & 0.2 & 0.2 \\
\hline \multicolumn{4}{|l|}{ ÍNDICES CUANTITATIVOS } \\
\hline Indice de diversidad interacciones (Shannon) & 3.68 & 2.97 & 2.82 \\
\hline Indice de equitatividad interacciones (Shannon) & 0.67 & 0.66 & 0.69 \\
\hline Indice de equitatividad interacciones (Alatalo) & 0.51 & 0.69 & 0.72 \\
\hline H2 (nivel de la especialización de la red) & 0.5 & 0.6 & 0.4 \\
\hline Fuerza de interacción asimétrica & 0.53 & 0.53 & 0.56 \\
\hline
\end{tabular}

Fase masculina (M), fase femenina (F) y capacidad de transporte de polen (P) de tres especies de Oenocarpus en la Amazonia colombiana.

Male phase (M).Female phase (F) and Pollen carrying capacity (P) of three species of Oenocarpus in the Colombian Amazon.

Especificidad: Los resultados de especificidad son consecuentes con las preferencias encontradas en el anterior análisis de la red. Las tres especies de palmas comparten pocas especies entre sí. Los valores más bajos de similaridad en la composición en fase masculina se presenta entre $O$. bataua y $O$. balickii con el $21 \%$ y entre $O$. bataua y $O$. minor con el 23\% de especies compartidas; las palmas que compartieron mayor número de visitantes fueron $O$. balickii y $O$. minor con el $51 \%$ de especies compartidas. En fase femenina, cerca de la mitad de las especies fueron compartidas entre palmas ya que los valores de similaridad oscilaron entre 45 y $57 \%$. Sin embargo, con respecto a las especies que llegaron cargadas de polen y lo depositaron en las flores femeninas se compartió entre el 38 y $53 \%$.
Los análisis de similitudes (ANOSIM) y el análisis de ordenamiento con la técnica de escalamiento multidimensional no métrico (NMSD) con los que se exploró la similaridad a partir de la abundancia, permiten pensar en la preferencia y, por lo tanto, la especificidad de los visitantes por cada palma. Aunque algunas de las especies se comparten, en cada fase la abundancia de los visitantes en cada palma determina el grado de asociación y por lo tanto de preferencia. La prueba ANOSIM indicó diferencias estadísticas significativas en la similaridad de visitantes en fase masculina $\mathrm{R}_{\mathrm{m}}=0.756(\mathrm{p}=0.005)$, fase femenina $\mathrm{R}_{\mathrm{f}}=0.861$ $(\mathrm{p}=0.005)$ y con los insectos polinizadores $\mathrm{R}_{\mathrm{p}}=0.811(\mathrm{p}=0.005)$. De igual manera, el ordenamiento diferencial de las especies en la gráfica (NMSD) mostró una clara separación 
A

Fase masculina

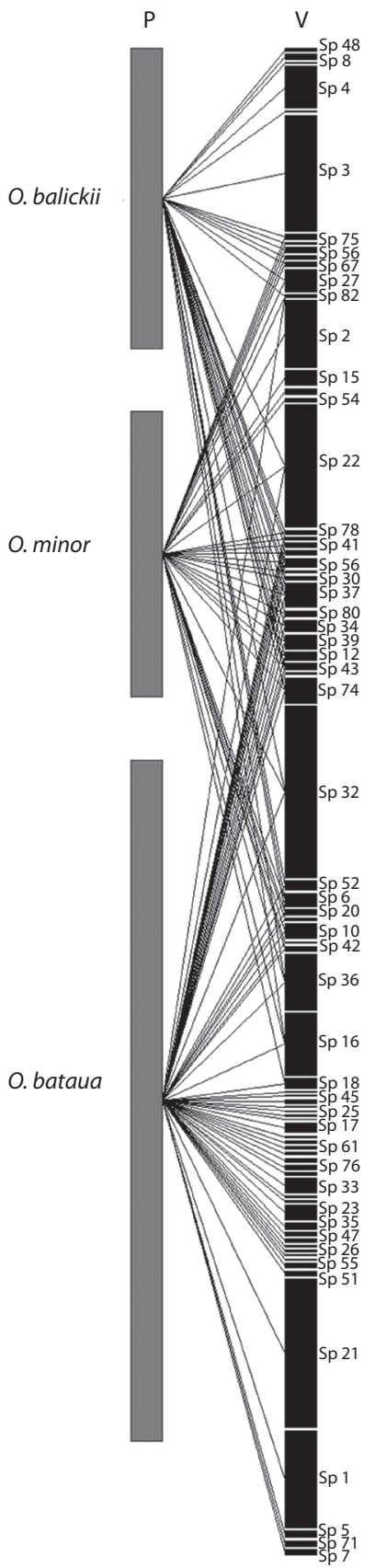

B

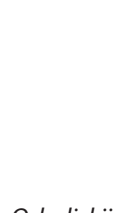

O. minor

O. bataua

.

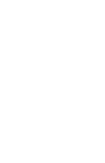

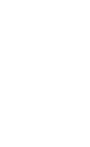

(2)

. v
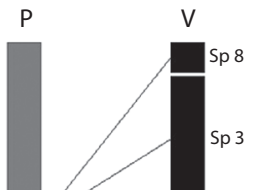

.
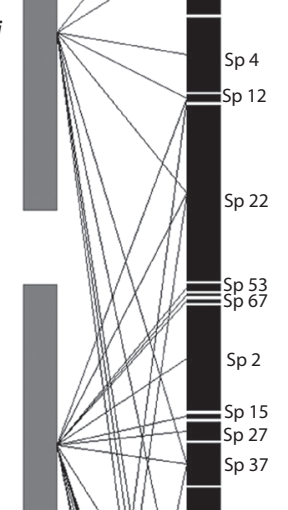

O. minor

O. balickii<smiles>[Te]</smiles>

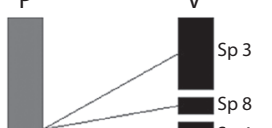

the

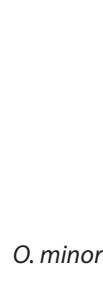

.
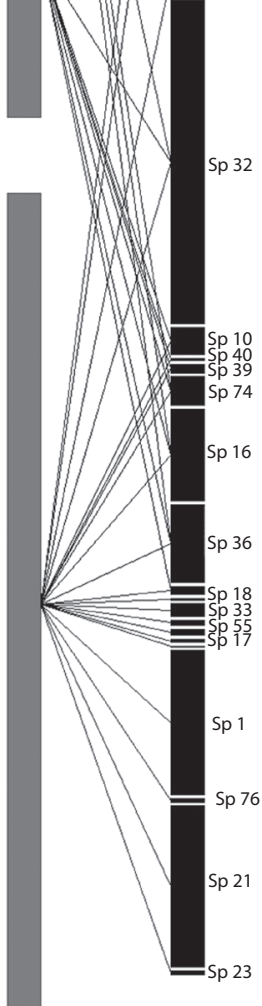

O. bataua
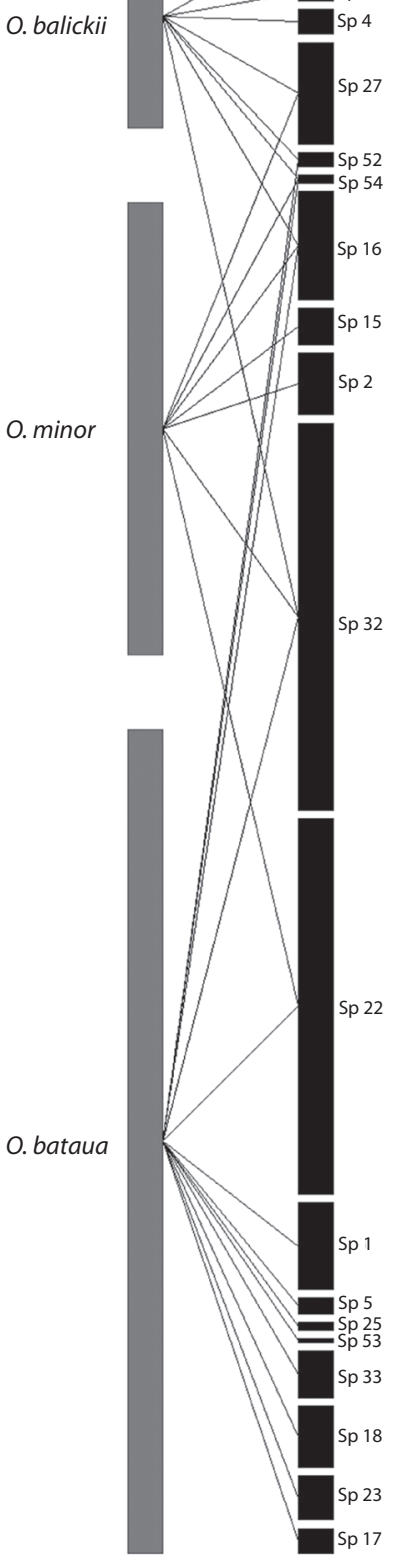

Fig. 2. Red bipartita para tres especies de Oenocarpus en la Amazonia colombiana. Las especies de palmas e insectos se representan por rectángulos y la interacción se representa por una línea que las conecta. A. Red de interacción en fase masculina, B. Red de interacción en fase femenina. C. Red de interacción de visitantes polinizadores (con cargas polínicas en su cuerpo).

Fig. 2. Bipartite networks of three Oenocarpus species in the Colombian Amazon. Palms and insects are represented by rectangles and the interaction is represented by a line connecting them. A. Network in male phase, B. Network in female phase. C. Network in pollinators (with pollen in their bodies). 


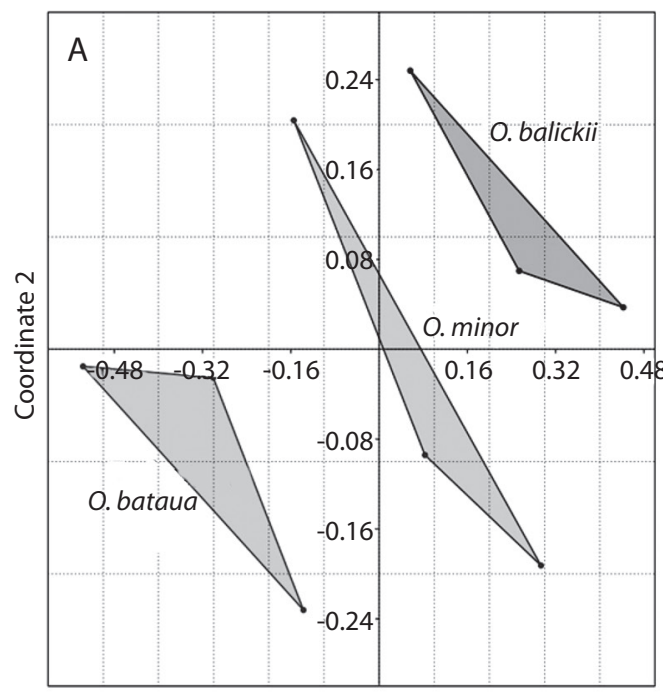

Coordinate 1

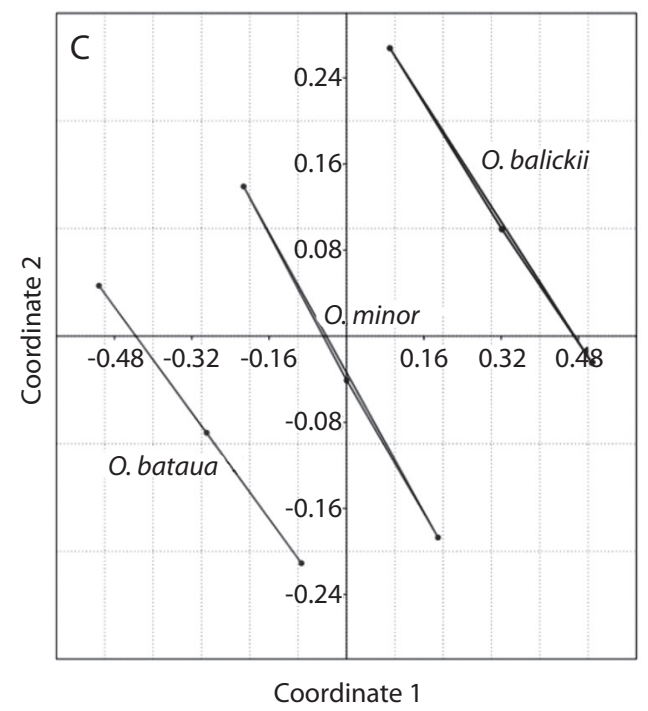

de los visitantes de cada palma (Fig. 3), lo que da prueba de la baja similaridad y preferencias de los visitantes por su palma hospedera respectiva.

Definición de polinizadores: Pocas especies participaron en la polinización de las tres especies de palmas; la mayoría de los visitantes florales no participaron (NP) en la transferencia de polen desde la fase masculina a la femenina (Cuadro 2); la mayoría de insectos centraron su

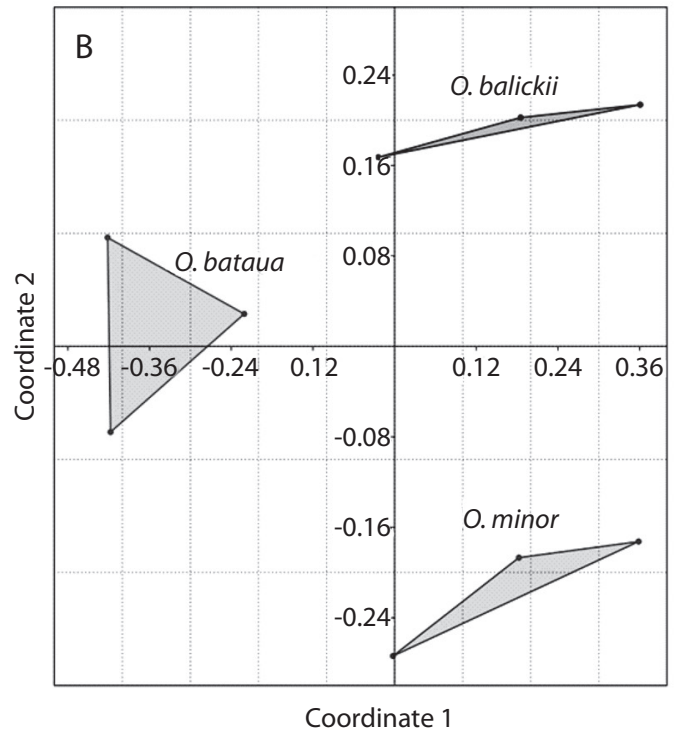

Fig. 3. Escalamiento Multidimensional No Métrico (NMSD) de los insectos visitantes de tres especies de Oenocarpus en la Amazonia colombiana. A. Inflorescencia en fase masculina, B. inflorescencia en fase femenina, C. Inflorescencia con polinizadores

Fig. 3. Nonmetric Multidimensional Scaling (NMSD) of visitors of three Oenocarpus speciesin the Colombian Amazon. A. Inflorescence in male phase, B. Inflorescence in female phase, C. Inflorescence with pollinators.

actividad y asociación en las fases masculinas de cada palma (Fig. 2). La mitad de los insectos que visitaron la fase masculina, lo hacieron en fase femenina, y de los insectos que llegaron en fase femenina tan sólo 19 especies llegaron cargadas con polen (Fig. 2). Las especies de visitantes que llegaron en fase femenina cargados de polen fueron coleópteros, principalmente curculiónidos de los géneros Derelomus, Phyllotrox, Anchyllorhynchus, Andranthobius, Terires y varias especies de Baridinae; Nitidúlidos del género Mystrops (Nitidulidae: Coleoptera) y ocasionalmente abejas del género Oxytrigona (Apidae: Himenoptera) (Cuadro 2). Aunque los géneros a los que pertenecen los insectos cargados de polen fueron comunes, cada palma fue visitada y polinizada por especies de coleópteros diferentes (Fig. 4). En cada palma se pueden encontrar varios polinizadores 
pero cada uno presenta un papel diferencial en la polinización (Fig. 4).

Éxito reproductivo: Las inflorescencias de las especies de Oenocarpus siguieron un mismo patrón: presentaron alta producción de flores femeninas, pero un número bajo de flores completaron su ciclo y llegaron a convertirse en frutos maduros para ser dispersados; las restantes fueron abortadas o murieron a lo largo del ciclo reproductivo. Una inflorescencia de $O$. balickii produjo en promedio 4105 $( \pm 1234 \mathrm{DE}, \mathrm{n}=10)$ flores femeninas, de las cuales tan sólo $43 \%$ se convirtieron en frutos; $O$. Bataua produjó en promedio $5736( \pm 1495 \mathrm{DE}$, $\mathrm{n}=10$ ) flores femeninas, de las cuales tan sólo $24.2 \%$ se convirtieron en frutos; y $O$. Minor produjo en promedio $3623( \pm 1296 \mathrm{DE}, \mathrm{n}=10)$ flores femeninas por inflorescencia, de las cuales tan sólo $25 \%$ se convirtieron en frutos.

\section{DISCUSIÓN}

Los resultados indican que las tres especies de Oenocarpusque crecen simpátricas en la Amazonia colombiana presentan un patrón reproductivo similar: al parecer producen inflorescencias a lo largo del año, las inflorescencias son iguales en su plan estructural, presentan semejanza en las fases reproductivas, la
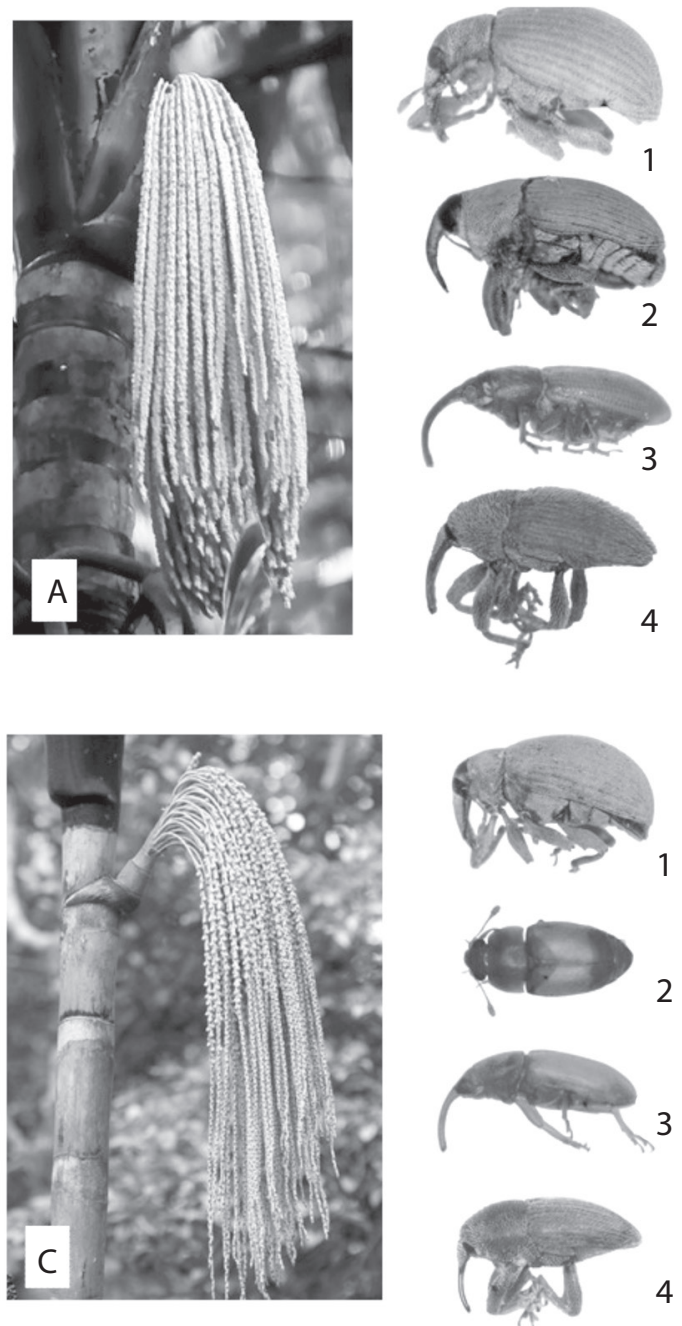
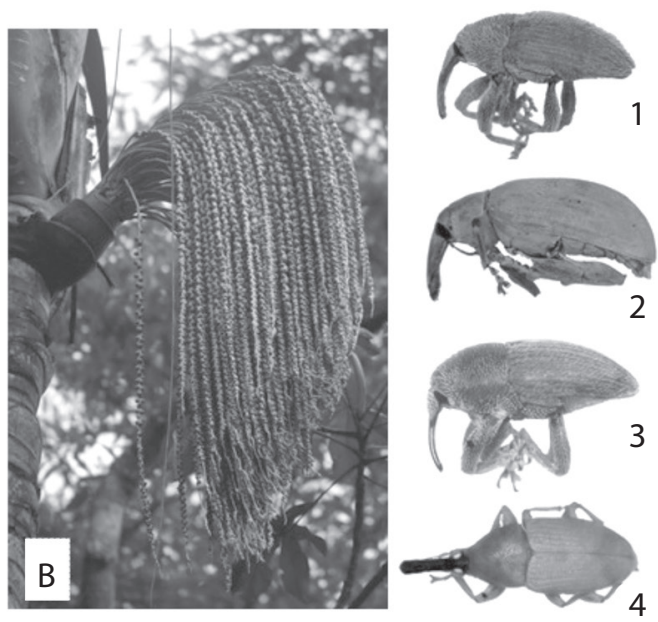

Fig. 4. Coleópteros polinizadores de especies de Oenocarpus. A. Oenocarpus balickii, B. O. batana y C. O. minor. Polinizadores indicados como: A1. Anchylorhynchus sp.1, A2. Limnonotus sp.1., A3. Phyllotrox sp.35 y A4. Baridinae Gen.4, sp.2. B1. Baridinae Gen.4, sp.1, B2. Anchylorhynchus tricarinatus, B3. Baridinae Gen.4, sp.2 y B4. Perelleschus sp.1, C1. Anchylorhynchus bicarinatus, C2. Mystrops salazari, C3. Phyllotrox sp.37 y C4. Baridinae Gen.4, sp.2.

Fig. 4. Beetle pollinators of Oenocarpus species. A. Oenocarpus balickii, B. O. bataua and C. O. minor. Pollinators indicated as: A1. Anchylorhynchus sp.1, A2.Limnonotus sp.1, A3. Phyllotrox sp.35 and A4. Baridinae Gen.4, sp.2. B1. Baridinae Gen.4, sp.1, B2. Anchylorhynchus tricarinatus, B3. Baridinae Gen.4, sp.2 and B4. Perelleschus sp.1, C1. Anchylorhynchus bicarinatus, C2. Mystrops salazari, C3. Phyllotrox sp.37 and C4. Baridinae Gen.4, sp.2. 
presentación del polen y la receptividad estigmática siguen el mismo ritmo, con igual longevidad y hora de actividad, y son polinizadas por grupos similares de insectos, principalmente coleópteros; sin embargo,un gran porcentaje de los polinizadores son específicos para cada especie. Este patrón reproductivo encontrado aquí, al parecer, lo presentan otras especies del género Oenocarpus (Cuadro 4), y parece ser constante a lo largo de la distribución de las especies, a juzgar por lo registrado en estudios previos sobre polinización de Oenocarpus realizados en diferentes localidades de Ecuador, Brasil y otras localidades de Colombia (Henderson, \& Furth, 1992; Küchmeister, Webber, Gottsberger, \& Silberbauer-Gottsberger, 1998; Núñez \& Rojas, 2008; Oliveira, Nazaré, \& Mota, 2002).

Se ha sugerido que cuando especies emparentadas coexistentes muestran un mismo patrón reproductivo (fenología, morfología, sistema reproductivo, biología floral y visitantes florales), suele presentarse una fuerte competencia por los polinizadores (Waser, 1983). Si tal competencia existe y se mantiene en el tiempo, se puede generar exclusión competitiva entre las especies competidoras (Sargent, \& Ackerly, 2008). La exclusión competitiva asume que las especies cercanas que mantienen un gran número de rasgos funcionales comunes no pueden coexistir, ya que las especies que mejor se apropien de los recursos o que interfieran en la apropiación de los mismos, excluirán competitivamente a sus parientes, generando patrones de repulsión filogenética dentro de las comunidades (Webb, Ackerly, McPeek, \& Donoghue 2002). Se esperaría entonces que las especies de Oenocarpus observadas ejercieran entre ellas exclusión competitiva. Sin embargo, esta no ocurre y la prueba de ello es que las tres especies se pueden encontrar creciendo entremezcladas y al parecer sin impedimentos

CUADRO 4

Aspectos reproductivos de especies de Oenocarpus encontrados en otros estudios

TABLE 4

Reproductive aspects in Oenocarpus species found in other studies

\begin{tabular}{|c|c|c|c|c|}
\hline & Oenocarpus bacaba & Oenocarpus minor & Oenocarpus bataua & Oenocarpus balickii \\
\hline Duración Fenología reproductiva & Todo el año & Todo el año & Todo el año & Todo el año \\
\hline Hora de antesis & Nocturna & Nocturna & Nocturna & Nocturna \\
\hline Duración antesis fase femenina & 5 días & 3 días & 4 días & 7 días \\
\hline Duración antesis fase masculina & 15 días & 7-10 días & 9 días & 15 días \\
\hline Dicogamia & protándrica & protándrica & protándrica & protándrica \\
\hline Polinización anemófila & No reportada & No reportada & No & No \\
\hline Recompensas florales & Polen & Polen & Polen & Polen \\
\hline $\begin{array}{l}\text { Termogénesis Inflorescencias } \\
\text { femeninas }{ }^{\circ} \mathrm{C}\end{array}$ & 13.2 & 9.2 & 3.4 & 5.5 \\
\hline $\begin{array}{l}\text { Termogénesis inflorescencias } \\
\text { masculinas }{ }^{\circ} \mathrm{C}\end{array}$ & 10 & 8.9 & 3.2 & 4.8 \\
\hline Aroma floral & Imitando frutos maduros & $\begin{array}{l}\alpha \text {-gurjuneno } \\
\text { ciclozativeno } \\
\text { trans-cariofileno }\end{array}$ & $\begin{array}{l}\text { Epibiciclosesqui } \\
\text { felandreno, limoneno } \\
\text { compuesto sin } \\
\text { identificar }\end{array}$ & $\begin{array}{l}\text { Imitando frutos } \\
\text { maduros }\end{array}$ \\
\hline Principal polinizador & $\begin{array}{l}\text { Mystrops y especies de } \\
\text { Curculionidae }\end{array}$ & Curculionidae & $\begin{array}{l}\text { Mystrops sp.1 Phyllotrox } \\
\text { sp.39 Anchylorhynchus } \\
\text { albidus }\end{array}$ & $\begin{array}{l}\text { Baridinae Gen. 4, sp. 1, } \\
\text { Phyllotrox sp. } 35\end{array}$ \\
\hline Referencia & Küchmeister et al.(1998) & $\begin{array}{l}\text { Küchmeister } \\
\text { et al.(1998) } \\
\text { Núñez (datos } \\
\text { sin publicar) }\end{array}$ & Núñez \& Rojas (2008) & Este estudio \\
\hline
\end{tabular}


reproductivos, a juzgar por el alto éxito reproductivo encontrado (valores entre 24 y $43 \%$ ), muy similar a lo registrado en especies de Oenocarpus creciendo en solitario (Núñez, \& Rojas, 2008).

La simpatría que presentan las especies de Oenocarpus se podría explicar por la existencia de aislamiento reproductivo entre ellas, aunque en plantas los impedimentos reproductivos pueden ocurrir antes de la polinización, e incluyen aislamiento temporal, espacial, mecánico y atracción diferencial de polinizadores (Grant, 1994). Los resultados encontrados en el presente estudio sugieren que en las especies de Oenocarpus el aislamiento reproductivo opera exclusivamente a través de la atracción y mantenimiento de polinizadores, ya que los otros tipos posibles de aislamiento son improbables para estas palmas: la floración continua en todas las especies descarta el aislamiento temporal; el aislamiento mecánico es poco probable debido a que no se presentan restricciones que impidan el libre acceso de los polinizadores hacia las flores de cualquiera de las palmas y aunque pudiese ocurrir aislamiento poscigótico entre combinaciones de especies, también hay fuerte evidencia de la posibilidad de entrecruzamiento que permite la hibridación entre pares de especies. La evidencia de la ocurrencia de hibridación entre especies de Oenocarpus ha sido registrada por Henderson et al. (1995) quienes mencionaron híbridos entre O. bataua-O.bacaba, O. bataua-O.minor y $O$. bacaba-O.minor.

El patrón de asociación mostró 79 especies de visitantes accediendo a las inflorescencias de las tres palmas, atraídos en fase masculina por gran cantidad de polen, algunos de ellos compartidos entre palmas, pero con pocos insectos en fase femenina, y la mayoría de ellos específicos a cada especie de palma. Los coleópteros de los géneros Derelomus, Phyllotrox, Anchyllorhynchus, Andranthobius, Terires, Baridinae (Curculionidae) y Mystrops (Nitidulidae), que fueron encontrados como los principales polinizadores de las especies de Oenocarpus, son insectos que viven dentro de las inflorescencias, sugiriendo una relación de mutua dependencia entre las palmas y los polinizadores. Tal especialización entre las especies de Oenocarpus y los coleópteros polinizadores se explica por la íntima asociación, ya que todas las especies de coleópteros de los géneros Derelomus, Phyllotrox, Anchyllorhynchus, Andranthobius, Terires, varias especies de Baridinae y Mystrops, que visitan y polinizan las tres palmas, dependen completamente de las inflorescencias que polinizan, dentro de las cuales tiene lugar su ciclo de vida. Por otro lado, las tres especies de palmas son también dependientes de los polinizadores debido a la xenogamia obligada que las caracteriza, de tal forma que se presenta una relación especializada de mutua dependencia. La dependencia obligada que tienen los coleópteros polinizadores por las inflorescencias y flores de diferentes especies de palmas ha sido ya documentada por varios autores (Henderson, 1986; Núñez, \& Carreño, 2013; Núñez et al., 2005; Núñez, \& Rojas, 2008). Sin embargo, en la mayoría de estudios no se han incluido observaciones detalladas que permitan dilucidar los factores que generan tal dependencia.

Una evidencia adicional para apoyar la hipótesis de la existencia de aislamiento reproductivo relacionado con mecanismos tendientes a la atracción de polinizadores específicos entre las especies de Oenocarpus, es la diferencia en la composición química del aroma floral. Núñez \& Rojas (2008) y Núñez (2007) encontraron que los aromas florales de O. bataua y de $O$. minor son altamente diferenciables, pues tan sólo comparten $22 \%$ de los compuestos entre ellos. La participación del aroma floral de cada palma en la atracción de polinizadores específicos en las tres especies estudiadasfue evidente a lo largo de la investigación, ya que siempre se presentó liberación de un fuerte e intenso aroma durante todo el ciclo floral en cada palma, con un aroma particular y fácilmente diferenciable para cada una de ellas. También y de manera coordinada, siempre coincidió la volatilización del aroma con el calentamiento interno de las inflorescencias, y la respuesta simultánea de los polinizadores, que llegarona las inflorescencias en los momentos en los que 
ocurrió la volatilización; en la fase femenina (que no ofrece ninguna recompensa) se produce aroma similar al de las flores masculinas, de tal forma que los polinizadores llegan atraídos por mimetismo químico, aspecto este registrado para un amplio grupo de palmas (Núñez, \& Rojas, 2008).

La especificidad del aroma entre especies de palmas coexistentes y congenéricas contribuye al establecimiento de palmas huésped fácilmente diferenciables por los polinizadores, debido a que el aroma genera mecanismos de atracción por las palmas y aprendizaje por parte de los insectos, generando la separación y preferencia de los insectos visitantes por especies de palmas exclusivas (Ervik et al., 1999; Knudsen, 1999; Knudsen, Andersson, \& Bergman, 1999).

Los coleópteros de los géneros Derelomus, Phyllotrox, Anchylorhynchus, Andranthobius, Terires, Baridinae (Curculionidae) y Mystrops (Nitidulidae) que fueron encontrados como los principales polinizadores de las especies de Oenocarpus, responden al aroma de las flores y a las características que les presenta cada palma, tal como se haencontrado a lo largo de la distribución de cada palma; por esta razón, han sido asociados, de manera exclusiva, con las inflorescencias de cada palma (Franz, \& Valente, 2005; Valente, \& Guimarães, 2010; Valente, \& Vanin, 2002). De tal manera que se podría pensar que las especies de cada grupo de estos coleópteros se han diversificado y especializado en grupos particulares de palmas, siempre manteniendo alta especificidad por las palmas que visitan.

Prueba de la alta diversidad pero también de alta especificidad de coleópteros Curculionidae asociados a flores de palmas la encontramos en los trabajos de Valente y Guimarães (2010) y Núñez (2014),en donde se compararon las especies de gorgojos asociados a 30 palmas en bosques brasileros y 20 especies de palmas en Colombia, respectivamente, con resultados similares y concluyentes en cuanto a la alta especificidad, con muy pocas especies de coleópteros compartidos entre palmas coexistentes, ya sea congenéricas o sin parentesco alguno.

El caso más evidente de asociación exclusiva entre curculiónidos y un grupo particular de palmas se ha documentado para especies de Anchylorhynchus. Este grupo de conformado por 45 especies, todas asociadas a flores de palmas de los géneros Oenocarpus, Syagrus y Butia, y en la mayoría de los casos, cada especie de coleóptero está asociado de manera exclusiva a una especie de palma en cada género (Medeiros, \& Núñez, 2013; Valente, \& Medeiros, 2013). Para las especies de Oenocarpus estudiadas aquíla especificidad e íntima asociación con especies de Anchylorhynchus también fue evidente: Anchylorhynchus tricarinatus asociado de manera exclusiva a flores de O. bataua, Anchylorhynchus bicarinatus asociado a flores de O. minor, y Anchylorhynchus sp.1 asociado a flores de $O$. balickii.

Estos casos en los que los coleópteros que polinizan y desarrollan su ciclo de vida dentro de las estructuras reproductivas de las plantas que polinizan han sido catalogados como sistemas especializados de polinización y se han registrado en un gran número de familias de plantas, además de las palmas. Algunas de las familias son Anonaceae (Gottsberger, 1999; Küchmeister et al., 1998; Küchmeister, Silberbauer-Gottsberger, \& Gottsberger, 1997), Cyclantaceae (Franz, 2005) y Araceae (Gibernau, Barabé, \& Labat, 2000).

Teniendo en cuenta que la principal importancia de las especies de Oenocarpus se encuentra en sus frutos, y que la producción de frutos se logra por la íntima asociación de las palmas con varias especies de coleópteros, es importante continuar con los estudios de las restantes especies de Oenocarpus y cuantificar la participación de los visitantes florales en otras localidades donde estas especies se encuentran. De esta manera se podría confirmar el alto grado de especialización que encontramos en las tresespecies de Oenocarpus estudiadas, información que sin lugar a dudas es fundamental para la conservación, los planes de manejo y aprovechamiento, y la futura domesticación de palmas con tan alto potencial. 
Aunque la similitud en la estrategia de reproducción encontrada en especies de Oenocarpus no es común a todas las palmas congénericas, v. gr., Geonoma (Borchsenius, 1997; Listabarth, 1993), se ha registrado para especies de Bactris (Henderson et al., 2000; Listabarth, 1996) y Phytelephas (Ervik et al., 1999). Por esta razón, estudios como el que aquí presentamos, en los que se abordan varias especies congenéricas y simpátricas proporcionan un mejor entendimiento de los mecanismos evolutivos de las estrategias de reproducción de grupos de especies emparentadas.

Debido a que en palmas son pocos los trabajos que evalúan conjuntos de especies simpátricas, congénericas o de estrecha relación filogenética, estamos muy lejos aún para definir el grado de homogeneidad y/o diferenciación en las estrategias de reproducción que presentan las especies que conforman los diferentes géneros de palmas, básicamente porque la mayoría de los trabajos consideran una o pocas especies, dejando a un lado los enfoques multiespecíficos que permitirían conocer realmente los patrones de reproducción de las especies de esta importante familia de plantas. En los estudios con especies individuales se aisla con frecuencia a la especie focal de su entorno comunitario en donde las otras especiespueden modificar el signo y el resultado de las interacciones con polinizadores. En la medida que se incrementen los estudios como el que aquí presentamos, involucrando varias especies congenéricas o de estrecha relación filogenética, se logrará definir el grado de homogeneidad y/o diferencias en las estrategias de reproducción de los diferentes géneros de palmas, claves para su aprovechamiento y conservación.

\section{AGRADECIMIENTOS}

Este estudio hace parte de la tesis de Doctorado, Programa en Ciencias-Biología, Universidad Nacional de Colombia de L. A. Núñez y C. Isaza. Recibimos financiación de Colciencias (proyecto No. 110148925263) y del programa marco FP-7 de la Unión Europea (Proyecto No. 21263). Agradecemos a los entomólogos Bruno de Medeiros, Diego Campos, Jhon Cesar Neita por la identificación de las especies. A la comunidad indígena en Amacayacu, Amazonas. A María Cristina Peñuela por ayuda y acceso a la Estación Biológica El Zafire de la Universidad Nacional sede Amazonia, y a Javier Carreño por su colaboración en la fase de campo.

\section{RESUMEN}

La comprensión de los mecanismos de polinización son de gran importancia para el desarrollo de planes de manejo y acciones de conservación de las especies de importancia económica. Con el objeto de conocer los mecanismos de polinización de palmas promisorias del género Oenocarpus (Arecaceae), se estudió la morfología, la biología floral, los visitantes, los polinizadores y éxito reproductivo de tres especies simpátricas en la Amazonía colombiana: O. bataua, O. balickii y O. minor. Durante el período 2010-2012 realizamos observaciones directas durante las fases de desarrollo de las inflorescencias en las tres especies. Se determinó la asociación de las palmas con sus visitantes florales a través de una red compleja, mientras que la especificidad o la preferencia de los insectos por cada palma individual se evaluó mediante el análisis similitud pareada, análisis de similitud (ANOSIM), un análisis de ordenación a partir del escalamiento multidimensional no métrico (NMSD). Las tres palmas florescen todo el año; sus inflorescencias contienen raquilas que cuelgan de un raquis corto, y presentan flores agrupadas en díadas o tríadas. Las inflorescencias son protandras, termogénicas; la antesis tiene lugar durante el día, pero la polinización es nocturna. Se registraron 79 especies de insectos, principalmente coleópteros, 33 de los cuales visitaron O. balickii, 63 a $O$. bataua y 33 a $O$. minor. Aunque compartían algunos visitantes, su abundancia en la fase pistilada, así como la carga de polen mostró que sólo unas pocas especies de Curculionidae y Nitidulidae son los polinizadores de las tres especies. Las diferencias en la estructura de la red entre las fases estaminadas y pistiladas, así como la diferencia en la abundancia encontrada con las pruebas de similitud ANOSIM y NMSD, sugieren una alta especificidad de polinizadores, lo que lleva a un aislamiento reproductivo entre las tres especies. Debido a que se encontró que todas las especies de coleópteros polinizadores desarrollan su ciclo de vida dentro de las inflorescencias de cada palma, se sugiere la presencia de un sistema especializado y de mutua dependencia entre los polinizadores y las especies de Oenocarpus. Esta especialización garantiza la disponibilidad de polinizadores durante todo el año.

Palabras clave: biología reproductiva, Coleoptera, producción de frutos, mutualismo, palmas tropicales. 


\section{REFERENCIAS}

Balick, M. J. (1992). Jessenia y Oenocarpus: Palmas aceiteras neotropicales dignas de ser domesticadas (Estudio FAO Producción y protección vegetal 88). Roma: FAO.

Balick, M. (1982). Palmas neotropicales nuevas fuentes de aceite comestible. Interciencia, 7(11), 25-29.

Barfod, A., Hagen, M., \& Borchsenius, F. (2011).Twentyfive years of progress in understanding pollination mechanisms in palms (Arecaceae). Annals of Botany, 108, 1503-1516.

Bernal, R., Galeano, G., \& Henderson, A. (1991). Notes on Oenocarpus (Palmae) in the Colombian Amazon. Brittonia, 43(3), 154-164.

Borchsenius, F. (1993). Flowering biology and insect visitation of three Ecuadorean Aiphanes species. Principes, 37, 139-150.

Borchsenius, F. (1997). Flowering biology of Geonoma irena and G. cuneata var. sodiroi (Arecaceae). Plant Systematics and Evolution, 208, 187-196.

Briggs, D., \& Walters, S. (1997). Plant variation and evolution. Cambridge, England: Cambridge University Press.

Brokamp, G., Valderrama, N., Mittelbach, M., Grandez, C., Barfod, A., \& Weigend, M. (2011). Trade in Palm Products in North-Western South America. Botanical Review, 77, 571-600.

Clarke, K., \& Gorley, R. (2001). PRIMER v5: User Manual Tutorial, Primer-e. Plymouth, United Kingdom: Plymouth Marine Laboratory.

Cox, T., \& Cox, M. 2001. Multidimensional scaling. United States of America: Chapman \& Hall/CRC.

Dafni, A. (1992). Pollination Ecology. Apractical approach. Oxford, England: Oxford University Press.

Dormann, C., Frund, J., Blutgeb, N., \& Gruber, B. (2009). Indices, graphs and null models: analyzing bipartite ecological networks. The Open ecology Journal, 2, 7-24.

Ervik, F., Tollsten, L., \& Knudsen, J. (1999). Floral scent chemistry and pollination ecology in phytelephantoid palms (Arecaceae). Plant Systematics and Evolution, 217, 279-297.

Fava, W., Covre, W., \& Sigrist, M. (2011). Attalea phalerata and Bactris glaucescens (Arecaceae, Arecoideae): Phenology and pollination ecology in the Pantanal, Brazil. Flora, 206, 575-584.

Franz, N. (2005). Towards a phylogenetic system of Derelomine flower weevils (Coleoptera: Curculionidae). Systematic Entomology, 31, 1-68.

Franz, N., \& Valente, R. (2005). Evolutionary trends in derelomine flower weevils: from associations to homology. Invertebrate Systematics, 19, 499-530.
Galeano, G., \& Bernal, R. (2010). Palmas de Colombia: Guía de campo. Bogotá, Colombia: Editorial Universidad Nacional de Colombia.

Galeano, G., Peñuela-Mora, M., \& Núñez, L. A. (2013). Guía de las palmas de la Estación Biológica El Zafire, Amazonas, Colombia. Universidad Nacional de Colombia, Sede Amazonia. Bogotá, Colombia: Impresos Panamericana.

Gibernau, M., Barabé, D., \& Labat, D. (2000). Flowering and pollination of Philodendron melinonii (Araceae) in French Guiana. Plant Biology, 2, 330-333.

Gottsberger, G. (1999). Pollination and evolution in neotropical Annonaceae. Plant Species Biology, 14, 143-152.

Grant, V. (1994). Modes and origins of mechanical and ethological isolation in angiosperms. Proceedings of the National Academy of Sciences of the United States of America, 91, 3-10.

Guimarães, P. R., Rico-Gray, J., Oliveira, V., Izzo, P., Reis, T., \& Thompson, T. (2007). Interaction intimacy affects structure and coevolutionary dynamics inmutualistic networks. Current Biology, 17, 1797-1803.

Hammer, O., Harper, D., \& Ryan, P. (2001). Paleontological statistics software package for education and data analysis. Paleontología Electrónica, 4, 1-9.

Henderson, A. (1986). A Review of pollination studies in the Palmae. Botanical Review, 52, 221-259.

Henderson, A. (2002). Evolution and ecology of palms. New York, United States of America: The New York Botanical Garden Press.

Henderson, A., \& Furth, D. (1992). Biología reproductiva de Jessenia y Oenocarpus. In M. J. Balick (Ed.), Jessenia y Oenocarpus: Palmas aceiteras neotropicales dignas de ser domesticadas (pp. 131-134). Roma, Italia: Estudio FAO Producción y protección vegetal 88.

Henderson, A., Galeano, G., \& Bernal, R. (1995). Field Guide to the Palms of the Americas. Princeton, New Jersey: Princeton Univesity Press.

Henderson, A., Pardini, A., Dos Santos Rebello, J., Vanin, S., \& Almeida, D. (2000). Pollination of Bactris (Palmae) in an Amazon forest. Brittonia, 52, 160-171.

Herrera, C. (2005). Plant generalization on pollinators: species property or local phenomenon? American Journal of Botany, 92, 13-20.

Kearns, C., \& Inouye, D. (1993). Techniques for pollination biologist. Niwot, Colorado: University Press of Colorado.

Knudsen, J. T. (1999). Floral scent chemistry in geonomid palms (Palmae: Geonomeae) and its importance in maintaining reproductive isolation. Memoirs of the New York Botanical Garden, 83, 141-168.

Knudsen, J., Andersson, S., \& Bergman, P. (1999). Floral scent attraction in Geonoma macrostachys, an 
understorey palm of the Amazonian rain forest. Oikos, 85, 409-418.

Küchmeister, H., Silberbauer-Gottsberger, I., \& Gottsberger, G. (1997). Flowering, pollination, nectar standing crop, and nectaries of Euterpe precatoria (Arecaceae), an Amazonian rain forest palm. Plant Systematics and Evolution, 206, 71-97.

Küchmeister, H., Webber, A., Gottsberger, G., \& Silberbauer-Gottsberger, I. (1998). A polinização e sua relação com a termogênese em espécies de Arecaceae e Annonaceae da Amazônia Central. Acta Amazonica, 28, 217-245.

Listabarth, C. (1993). Pollination in Geonoma macrostachys and three congeners, G. acaulis, G. gracilis and G. interrupta. Botanica Acta, 106, 496-506.

Listabarth, C. (1996). Pollination of Bactris by Phyllotrox and Epurea. Implications of the palm breeding beetles on pollination at the community level. Biotropica, $28,69-81$.

Macía, M., Armesilla, P., Cámara-Leret, R., PaniaguaZambrana, N., Villalba, S., Balslev, H., \& PardoDe-Santayana, M. (2011). Palm uses in northwestern South America: A quantitative review. The Botanical Review, 77, 462-570.

Medeiros, B., \& Nuñez, L. A. (2013). Three new species of Anchylorhynchus Schoenherr, 1836 from Colombia. Zootaxa, 3636(2), 394-400. doi: http://dx.doi. org/10.11646/zootaxa.3636.2.10

Miranda, J., Montaño, F., Zenteno, F., Nina, H., \& Mercado, J. (2008). El Majo (Oenocarpus bataua): Una alternativa al biocomerico en Bolivia. La Paz, Bolivia: Trópico-PNBS-FAN.

Núñez, L. A. (2007). Evaluación del aislamiento reproductivo entre tres especies simpátricas Oenocarpus bataua, Oenocarpus minor y Euterpe precatoria (Palmae: Arecoideae: Euterpeae) en un bosque de los Andes en Colombia (Tesis de Maestría). Universidad Nacional de Colombia. Bogotá, Colombia.

Nuñez, L. A., \& Carreño, J. (2013). Biología reproductiva de Mauritia flexuosa en Casanare, Orinoquia colombiana. In C. A. Lasso, A. Rial, \& V. González (Eds.), Morichales y Cananguchales de la Orinoquia y Amazonia (Colombia-Venezuela) (p.p. 119-150). Bogotá D.C., Colombia: Serie Editorial Recursos Hidrobiológicos y Pesqueros Continentales de Colombia. Instituto de Investigación de Recursos Biológicos Alexander von Humboldt.

Núñez, L. A., Bernal, R., \& Knudsen, J. (2005). Diurnal palm pollination by mytropine beetles: is it weather-related? Plant Systematics and Evolution, 208, 187-196.
Núñez, L. A. (2014). Patrones de asociación entre polinizadores y palmas en Colombia. Con énfasis en palmas de importancia económica (Tesis Doctoral). Universidad Nacional de Colombia. Bogotá, Colombia.

Núñez, L. A., \& Rojas, R. (2008). Biología reproductiva y ecología de la polinización de la palma milpesos Oenocarpus bataua en los Andes colombianos. Caldasia, 30(1), 99-122.

Oliveira, M., Nazaré, R., \& Mota, M. (2002). Estudo comparativo da qualidade do palmito de bacabinha com $o$ do açaizeiro (Comunicado Técnico, 39). Belém, Brazil: Embrapa Amazônia Oriental.

Pellmyr, O. (2002). Pollination by animals. In C. M. Herrera, \& O. Pellmyr (Eds), Plant-animal interactions: an evolutionaryapproach (pp. 157-184). Oxford, England: Blackwell Science Publishing.

Sargent, R., \& Ackerly, D. (2008). Plant-pollinator interactions and the assembly of plant communities. Trends in Ecology and Evolution, 23, 123-130.

Valente, R., \& De Medeiros, B. (2013). A new species of Anchylorhynchus Schoenherr (Coleoptera: Curculionidae) from the Amazon, with a record of a new host palm for the genus. Zootaxa, 3709(4), 394-400. doi: http://dx.doi.org/10.11646/zootaxa.3709.4.6

Valente, R., \& Vanin, S. A. (2002). Curculionidae (Coleoptera) em inflorescência de Attalea maripa (Aubl.) Mart. (Arecaceae). In P. L. B. Lisboa (Ed.), Caxiuana População tradicionais, Meio Fisico e Diversidade biológica (pp. 483-502). Belém-Brazil: Museu Paraense Emílio Goeld.

Valente, R. M., \& Guimarães, J. R. R. (2010). Os besouros gorgulhos de flores de palmeiras. In A. C. M. Oliveira, J. B. Santos, \& M. C. Santos-Costa (Eds), Os animais da Tanguro, Mato Grosso:diversidade na zona de transição entre a floresta amazônica e o cerrado (pp. 26-30). Belém, Brazil: Museu Paraense Emílio Goeldi.

Vásquez, D., \& Aizen, M. (2004). Asymmetric specialization: a pervasive feature of plant-pollinator interactions. Ecology, 85, 1251-1257.

Waser, N. (1983). Competition for pollination and floral character differences among sympatric plant species: a review of evidence. In C. E. Jones, \& R. J. Little (Eds.). Handbook of experimental pollination biology (pp. 277-293). New York, United States of America: Van Nostrand Reinhold.

Webb, C., Ackerly, D., McPeek, M., \& Donoghue, M. (2002). Phylogenies and community ecology. Annual Review of Ecology and Systematics, 33, 475-505.

Zona, S., \& Henderson, A. (1989). A review of animalmediated seed dispersal of palms. Selbyana, 11, 6-21. 
